\title{
Some remarks on the 14th problem of Hilbert
}

\author{
By \\ Masayoshi NAGATA and Kayo OTSUKA
}

(Received September 27, 1965)

As for the 14th problem of Hilbert, we know that the answer is negative (see [2]). But there are several known sufficient conditions for the affirmative answer (those in [6] and [8] are important). In the present paper, we are to give some sufficient conditions of a new type but related to the one given in [4]. We shall treat the problem in a generalized form. Namely, we consider a pseudo-geometric integral domain $K$, satisfying two conditions $(K, 1)$ and $(K, 2)$ below, as a ground ring. ${ }^{1)}$

$(K, 1)$ The altitude formula holds for $K$.

$(K, 2)$ Normal spots over $K$ are analytically irreducible.

Let $A_{1}, \cdots, A_{n}$ be normal affine rings over $K$ and let $A$ be the direct sum of them. Let $R$ be an integral domain containing $K$ and contained in $A$ such that (i) the field of quotients $Q(R)$ of $R$ is a subring of the total quotient ring of $A$ (i.e., the natural homomorphism from $R$ into $A_{i}$ (given by multiplying the identity of $A_{i}$ ) is injective (=isomorphism into)) and (ii) $R=A \cap Q(R)$. Our aim is to give some sufficient conditions for this ring $R$ to be an affine ring over $K$. In our treatment, we assume one more condition for

1) As for the words pseudo-geometric, altitude formula, affine rings, height, depth and so on, they are understood as those in our book [5]. As for these conditions $(K, 1)$ and $(K, 2)$, we may start with the derived normal ring of $K$ instead of $K$ itself. Then $(K, 1)$ follows from $(K, 2)$ (See [3]). Therefore it is really enough ito assume $(K, 2)$ only. Spot $=$ locality. 
$R$. Namely, we assume that for every prime ideal $\mathfrak{p}$ of $R$, there is a prime ideal $\mathfrak{p}^{\prime}$ of $A$ which lies over $\mathfrak{p}$. Then our main results. can be stated as follows:

Theorem 1. If $\mathfrak{m} R_{\mathfrak{m}}$ has a finite basis for every maximal. ideal $\mathfrak{m}$ of $R$, then $R$ is finitely generated over $K$.

Theorem 2. Assume that for every pair of maximal ideal $\mathfrak{m}$ and a prime ideal $\mathfrak{p}$ of height 1 in $R$ such that $\mathfrak{p} \subseteq \mathfrak{m}$, the maximal ideal $\mathfrak{m} R_{\mathfrak{m}} / \mathfrak{p} R_{\mathfrak{m}}$ has a finite basis and that every normal spot over $K$ contains a prime element unless the spot is a field, ${ }^{2)}$ then $R$ is finitely generated over $K$.

We shall show also in this paper that the main theorem in [4] can be simplified if the ground rings are restricted to such one in. the present paper.

\section{§1. The main theorems.}

We shall make use of the following lemma of Zariski [7]: ${ }^{3)}$

Lemma 1. If a normal spot $P$ over $K$ is dominated by. another spot $Q$ over $K$, then $P$ is a subspace of $Q$ (under their natural topologies).

Now we shall prove Theorem 1 . So, we assume that $\mathfrak{m} R_{\mathfrak{m}}$ has a finite basis for every maximal ideal $n$ of $R$. Since $A_{i}$ are normal rings and since $R=A \cap Q(R)$, there is a normal affine ring $B$ over $K$ with an ideal $\mathfrak{a}$ such that $R$ is the a-transform $T(\mathfrak{a})$ of $B$ (see [4]). Assume for a moment that $R$ is not finitely gederated over $K$. Then, as was shown in the proof of Theorem 4 in [1] or in that of Lemma 2.7 in [4], there is a sequence of normal affine rigns. $B=B_{0} \subset B_{1} \subset \cdots \subset B_{r} \subset \cdots$ with ideals $a \subset a_{1} \subset \cdots \subset a_{r} \subset \cdots$ such that (1) $R=\cup_{i} B_{i}$ and (2) each $a_{i}$ is different from $B_{i}$ and is the intersection of prime ideals of height 1 in $B_{i}$ which contains $a$. Let $a^{*}$ be

2) This second condition is satisfied by fields.

3) Though Zariski stated this result for spots over a field, his Theorem 1 in [7] is good for the general case and we have no difficulty in proving this lemma. 
the union of all $\mathfrak{a}_{i}$ and let $\mathfrak{m}$ be a miximal ideal of $R$ containing $\mathfrak{a}^{*}$. Since $\mathfrak{m} R_{\mathfrak{m}}$ has a finite basis, $B$ may be replaced by one $B_{i}$ which contains a basis for $\mathfrak{m} R_{\mathfrak{m}}$. Thus we may assume that $\mathfrak{m}^{\prime \prime}=$ $\mathfrak{m} \cap B$ generates $\mathfrak{m} R_{\mathfrak{m}}$ (in $R_{\mathfrak{m}}$ ). Since there is a prime ideal $\mathfrak{m}^{\prime}$ of $A$ which lies over $\mathfrak{m}$, we see that $R / \mathfrak{m}$ is finite algebraic over $B / \mathfrak{m}^{\prime \prime}$. Now we consider the rings $B_{\mathfrak{m}^{\prime \prime}}, R_{\mathfrak{m}}$ and $A_{\mathfrak{m}^{\prime}} . \quad B_{\mathfrak{m}^{\prime \prime}}$ and $A_{\mathfrak{m}^{\prime}}$ are normal spots over $K$, and therefore $B_{\mathfrak{m}^{\prime \prime}}$ is a subspace of $A_{\mathfrak{m}}$ ' Since $R_{\mathfrak{m}}$ is in between of them, we see that $R_{\mathfrak{m}}$ is a local ring which may not be Noetherian and $B_{\mathfrak{m}}{ }^{\prime \prime}$ is a subspace of $R_{\mathfrak{m}}$. Namely, the completion $\left(B_{\mathfrak{m}}^{\prime \prime}\right)^{*}$ of $B_{\mathfrak{m}^{\prime \prime}}$ can be regarded as a subring of the completion $\left(R_{\mathfrak{m}}\right)^{*}$ of $R_{\mathfrak{m}}$. Since $\mathfrak{m}^{\prime \prime}$ generates $\mathfrak{m} R_{\mathfrak{m}}$ and since $R / \mathfrak{m}$ is finite algebraic over $B / \mathfrak{m}^{\prime \prime}$, we see that $\left(R_{\mathfrak{m}}\right)^{*}$ is integral over $\left(B_{\mathfrak{m}^{\prime \prime}}\right)^{*}$. Since $R_{\mathfrak{m}}$ is contained in the field of quotients of $B$, it follows now that $R_{\mathfrak{m}}$ is integral over $B_{\mathfrak{m}^{\prime \prime}}$ and therefore $R_{\mathfrak{n l}}=B_{\mathfrak{m}^{\prime \prime}}$ (see [5] (37.4)). This contradicts to the infiniteness of the sequence $B \subset B_{1} \subset \cdots \subset B_{r} \subset \cdots$ and the proof of Theorem 1 is completed.

Now we shall prove Theorem 2. By virtue of Theorem 1, we have only to show that $m R_{\mathfrak{m}}$ has a finite basis for every maximal ideal $\mathfrak{m}$ of $R$. Take a normal affine ring $B$ with an ideal a such that $R$ is the a-transform $T(\mathfrak{a})$ of $B$ and set $\mathfrak{n}^{\prime \prime}=\mathfrak{m} \cap B$. Then $B_{\mathfrak{m}^{\prime \prime}}$ is a normal spot, hence it has a prime element, say $p$. Then $p$ is a prime element in $R_{\mathfrak{n}}$. Therefore our assumption say that $\mathfrak{m} R_{\mathfrak{m}} / p R_{\mathfrak{m}}$ has a finite basis, which implies that $\mathfrak{m} R_{\mathfrak{m}}$ had a finite basis. This completes the proof of Theorem 2 .

\section{§. Supplementary remarks.}

(1) On the proof of the main theorem of [4]. The main theorem of [4] is as follows:

Let $K$ be a pseudo-geometric ring and let $A$ be a finitely generated ring over $K$. If a ring $R$ which is in between $K$ and $A$ is strongly submersive in $A$, then $R_{\mathrm{red}}=R /($ the radical of $R$ ) 
is a finite K-algebra.

What we want to remark here is that if $K$ is such one as in $\S 1$, and if furthermore $K$ satisfies the condition that every normal spot over $K$ has a semi-prime element unless the spot is a field, then the assertion can be proved in much simpler way.

In deed, it is easy to reduce the assertion to the case as in the begining of this paper. Take $B$ and $a$ as in the proof of Theorem 2 , and we take a semi-prime element $s$ of $B_{\mathfrak{m}^{\prime \prime}}$. Then $s$ is semiprime in $R_{\mathfrak{m}}$ and $R_{\mathfrak{n}} / s R_{\mathfrak{m}}$. is a subdirect sum of a finite number of Noetherian rings (using induction argument on height $\mathfrak{m}$ ), hence is Noetherian. Therefore $\mathfrak{m} R_{\mathfrak{m}}$ has a finite basis, and $R$ is a finite $K$ algebra. One should note that in this case, among the preliminary results in $\$ 1$ of [4], we need only Lemma 1.2 which asserts that if $R$ is strongly submersive in $A$ and if $a$ is an ideal of $R$, then $R / a$ is strongly submersive in $A / \mathfrak{a} A$.

By the way, we like to note here that the above mentioned proof really yields

Theorem $2^{*}$. Let $K$ and $R$ be as in the beginning of this paper. If, for every pair of maximal ideal $m$ of $R$ and a prime ideal $\mathfrak{p}$ of height 1 in $R$ contained in $\mathrm{m}$, the ring $R_{\mathfrak{m}} / \mathfrak{p} R_{\mathfrak{n}}$ is Noetherian and if every normai spot over $K$ has a semi-prime element unless the spot is a field, then it follows that $R$ is finitely generated over $K$.

(2) On the height of prime ideals of $R$.

For an integral domain I, we consider the following chain condition: ${ }^{4)}$

(C) If $\mathfrak{p}$ is a prime ideal of $I$, then every descending chain of prime ideals in $I$ which begins with $\mathfrak{p}$ and ends with 0 can be refined so that its length is equal to the height of $\mathfrak{p}$.

We begin with an easy

4) One can show easily that the chain condition (C) is satisfied by $K$ in the beginning of the present paper. 
Lemma 2. Let I be a Noetherian integral domain for which the altitude formula and the chain condition (C) hold good. Then these conditions hold for every affine ring over $I$.

Since the proof is easy, we omit it.

Now we come to the main remark:

Theorem 3. Let I be a Noetherian integral domain for which the altitude formula and the chain condition $(C)$ hold. Let $A$ be an affine ring over $I$ and let $R$ be a subring of $A$ which contains $K$. If $\mathfrak{p}$ is a prime ideal of $R$ for which there is a prime ideal $\mathfrak{p}^{\prime}$ of $A$ which lies over $\mathfrak{p}$, then

height $\mathfrak{p}+$ trans. $\operatorname{deg}_{I /(\mathfrak{p} \cap l)} R / \mathfrak{p}=$ height $(\mathfrak{p} \cap I)+$ trans. $\operatorname{deg}_{l} R$.

Proof. If $\mathfrak{p}=0$, then the aesertion is obvious, and we use induction argument on height $\mathfrak{p}$. Let $I^{*}$ be an afflne ring over $I$ which is contained in $R$. Then, by the altitude formula applied to $\mathfrak{p}^{*}=\mathfrak{p} \cap I^{*}$, we have

height $\mathfrak{p}^{*}+$ trans. $\operatorname{deg}{ }_{I /(\mathfrak{p} \cap I)} I^{*} / \mathfrak{p}^{*}=$ height $(\mathfrak{p} \cap I)+$ trans. $\operatorname{deg}_{I} I^{*}$.

Therefore the assertion is equivalent to the formula for $I^{*}$ instead of $I$. Therefore $I$ may be replaced by any of such $I^{*}$ by virtue of Lemma 2. In particular, we may assume that $R / \mathfrak{p}$ is algebraic over $I /(\mathfrak{p} \cap I)$. Considering $I_{(\mathfrak{p} \cap l)}$ instead of $I$, we may assume that $\mathfrak{p} \cap I$ is the unique maximal ideal of $I$. Now, let $\mathfrak{P}$ be the set of prime ideals of $A$ which lie over $\mathfrak{p}$. We may assume that $\mathfrak{p}^{\prime}$ is maximal in $\mathfrak{P}$. Since $R / \mathfrak{p}$ is a field by our assumption and since $A / \mathfrak{p}^{\prime}$ is an affine ring over the field $R / \mathfrak{p}$, we see that $\mathfrak{p}^{\prime}$ is a maximal ideal of $A$ and $A / \mathfrak{p}^{\prime}$ is algebraic over the field $R / \mathfrak{p}$. Let $\mathfrak{q}^{\prime}$ be a prime ideal of $A$ such that (i) $\mathfrak{q}^{\prime} \subset \mathfrak{p}^{\prime}$. (ii) depth $\mathfrak{q}^{\prime}=1$ and (iii) $\mathfrak{p} A \nsubseteq q^{\prime}$. Set $\mathfrak{q}=\mathfrak{q}^{\prime} \cap R$.

Case 1. Assume that $\mathfrak{q} \cap I=\mathfrak{p} \cap I$. Then, since $A / \mathfrak{q}^{\prime}$ is an affine ring over the field $I /(\mathfrak{p} \cap I)$, we see that trans. $\operatorname{deg}_{I(\mathfrak{p} \cap I)} A / \mathfrak{q}^{\prime}=1$. 
Since $R / \mathfrak{q}$ is a subring of $A / \mathfrak{q}^{\prime}$ and since $\mathfrak{q} \neq \mathfrak{p}$, we see that trans. $\operatorname{deg}_{I /(\mathfrak{P} \cap I)} R / \mathfrak{q}=1$. Now, by induction assumption, we have: height: $\mathfrak{q}+$ trans. $\operatorname{deg}_{I /(\mathfrak{p} \cap l)} R / \mathfrak{q}=$ height $(\mathfrak{p} \cap I)+$ trans. $\operatorname{deg}_{l} R$. Since height $\mathfrak{p}$. $\geq$ (height $\mathfrak{q})+1$, we see that height $\mathfrak{p}+$ trans. $\operatorname{deg}_{I /(\mathfrak{p} \cap I)} R / \mathfrak{p} \geq$ height $(\mathfrak{p} \cap I)+\operatorname{trans} . \operatorname{deg}_{l} R$. The converse inequality holds obviously because $I$ is Noetherian and we settle this case.

Case 2. Assume that $\mathfrak{q} \cap I \neq \mathfrak{p} \cap I$. Since $A / \mathfrak{q}^{\prime}$ is an affine ring over the ring $I /(\mathfrak{q} \cap I)$ and since $\mathfrak{p}^{\prime} / \mathfrak{q}^{\prime}$ is a maximal ideal of height 1 which lies over the maximal ideal $(\mathfrak{p} \cap I) /(\mathfrak{q} \cap I)$, we see that $A / \mathfrak{q}^{\prime}$ is algebraic over $I /(\mathfrak{q} \cap I)$ and that $(\mathfrak{p} \cap I) /(\mathfrak{q} \cap I)$ is of height 1. By the chain condition (C), we see that height $(\mathfrak{p} \cap I)=1+$ theight $(\mathfrak{q} \cap I)$, and therefore we settle this case similarly as in Case 1 above. Thus the proof of Theorem 3 is completed.

\section{References}

[1] M. Nagata, A treatise on the 14th problem of Hilbert, Mem. Kyoto Univ. Ser. A Math. 30 (1956-57), pp. 57-70; Correction and addition, ibid. pp. 197-200.

[2] - On the 14th problem of Hilbert, Amer. J. Math. 81 (1951), pp. 766772.

[3] - Note on a chain condition for prime ideals, Mem. Kyoto Univ. Ser. A Math. 32 (1959-60), pp. 85-90.

[4] - A thorem on finite generation of a ring, to appear in Nagoya. Math. J..

[5] Local rings, John Wiley, New York (1962).

[6] H. Weyl, Classical groups Princeton Univ. Press (1939).

[7] O. Zariski, A simple analytical proof of a fundamental property of birational transformations, Proc. Nat. Acad. Sci. U.S.A. 35 (1949), pp. 62-66.

[8] - Interprétationes algébrico-géométriques du quatorzième problème de Hilbert, Bull. Sci. Math. 78 (1954), pp. 155-168. 